\title{
Trans* und Substanzgebrauch: Bedingungen und Behandlungsempfehlungen
}

\section{Trans* People and Substance Abuse: Conditions and Recommendations for Treatment}

\author{
Autor \\ Gisela Wolf
}

Institut

Psychotherapeutische Praxisgemeinschaft, Berlin

\section{Schlüsselwörter}

Trans*, Substanzgebrauch, Diskriminierung, Gewalt, affirmative Suchttherapie

Key words

trans* people, substance-use, discrimination, violence, affirmative therapy

Bibliografie

DOI https://doi.org/10.1055/a-0715-1084

Online-Publikation: 14.9.2018

Suchttherapie 2018; 19: 186-192

(C) Georg Thieme Verlag KG Stuttgart · New York ISSN 1439-9903

Korrespondenzadresse

Dr. Gisela Wolf

Psychotherapeutische Praxisgemeinschaft

Neue Kantstraße 3

14057 Berlin

gisela.wolf.fr@googlemail.com

\section{ZUSAMMENFASSUNG}

Die von trans* Personen erlittenen Diskriminierungen und Gewalterfahrungen stellen maßgebliche Bedingungsfaktoren von schädlichem Substanzgebrauch dar. In dem vorliegenden Text werden strukturelle und manifeste Diskriminierungen und Gewalt aufgezeigt, mit denen sich trans* Personen auseinandersetzen müssen. Es werden Modelle referiert, die die Zusammenhänge zwischen gesellschaftlicher Ausgrenzung und Substanzgebrauch erfassbar machen und Empfehlungen für die Behandlung von trans* Personen in suchttherapeutischen Einrichtungen entwickelt.

\section{ABSTRACT}

Experiences with discrimination and violence are decisive conditions of substance abuse in the trans community. The article describes forms of discriminations and violence trans* people have to deal with. Theoretical concepts for conceptualization of the connections between stigmatization and substance-use are discussed. Recommendations for substance-abuse-treatment of trans* clients are derived.

\section{Einleitung}

Ich möchte die Leser*1 innen zunächst einladen, der Frage nachzugehen, was sie heute bereits alles getan haben, um von anderen als „Frau“ oder als „Mann“ gesehen werden zu können:

Wie haben Sie sich gekleidet, gesprochen, wie haben Sie sich in der Öffentlichkeit bewegt, wie sich mit Ihrer Gesundheit auseinandergesetzt? Was lief dabei routiniert und unwillkürlich ab? Was hat Mühe und Aufwand bereitet? Sind Sie heute bereits wegen etwas, was mit Ihrer Geschlechtlichkeit, mit Ihrem Frau- oder Mannsein zu tun hat, diskriminiert worden? Haben Sie Menschen im Unklaren über Ihre Geschlechtlichkeit gelassen? Wenn ja, wie haben diese reagiert? Wenn nein, überlegen Sie, warum es Ihnen wichtig gewesen ist, bezogen auf Ihre Geschlechtlichkeit eindeutig erkennbar aufzutreten.
Eine Reflexion dieser Erfahrungen öffnet die Tür zu diesem Text. Menschen, die sich als Trans* oder trans* definieren, bewegen und positionieren sich auf dem Kontinuum zwischen „Weiblichkeit“ und

1 Der hochgestellte Stern an der Stelle, wo sonst in der deutschen Sprache bruchlos eine geschlechterkonnotierte Endung an ein Substantiv angehängt wird, drückt aus, dass hier Menschen verschiedener Geschlechter benannt und sichtbar werden sollen. In der gesprochenen Sprache wird der Genderstern wie auch der Gendergap [„Leser_innen“] durch eine kleine Pause ausgedrückt. Am Ende eines Wortes gesetzt macht der hochgestellte Stern deutlich, dass an das Wort verschiedene Erweiterungen angefügt werden können. So werden beim Begriff „Trans*“ verschiedene mögliche Erweiterungen des Begriffs mitgedacht z. B. Transgender, Transsexuelle, Transidente ... [vergl. 1]. Andere Autor*innen beschreiben mit dem Oberbegriff „Trans*“ oder „trans*“ auch zusätzlich Personen, die sich auf einem Kontinuum der Geschlechtsidentitäten zwischen „männlich“ und "weiblich“ befindlich oder sich als sowohl „weiblich“ als auch „männlich“ definieren oder sich als außerhalb eines binär aufgespannten Geschlechterkontinuums stehend wahrnehmen [vergl. z.B. 2]. 
„Männlichkeit“ anders, als es den normativen geschlechtlichen Zuschreibungen an sie entspricht. Normative geschlechtliche Zuweisungen gehen bspw. davon aus, dass „Frauen“ dauerhaft einen aufgrund primärer und sekundärer Geschlechtsmerkmale eindeutig „weiblichen“ Körper aufweisen. Die Zuschreibung, dass dieser Körper „weiblich“ sei, erfolgt vielfach bereits vorgeburtlich und wird spätestens nach der Geburt auch amtlich, d. h. in die Geburtsurkunde eingetragen. Daraufhin konstruiert die Umwelt ihre Erwartungen an das Kind entsprechend und das Kind wird als „Mädchen“ zur „Frau“ sozialisiert und entsprechend angesprochen (mit weiblichem Namen und Pronomen). Es wird von ihr erwartet, dass sich ihre Geschlechtsidentität eindeutig „weiblich“ entwickelt, dass sie ihren Habitus täglich so gestaltet, dass keine Verwirrung bezogen auf ihre rasche und eindeutige Geschlechtereinordnung besteht. Solche geschlechterbezogenen Erwartungen bezeichnen wir als „ciszentristisch“, weil darin die Annahme, dass das Zuweisungsgeschlecht dem Identitätsgeschlecht lebenslang entspricht, als zentrale Norm gesetzt wird. Eine Person, die ihr Leben im Einklang mit diesen Normen lebt, wird als „cis-ident“ benannt. Lebt sie heterosexuell, dann wird ihre Partnerwahl bezogen auf eine als „gegengeschlechtlich“ eingeordnete Person als Affirmation ihrer Geschlechtsidentität gesehen und in einer heterozentristisch organisierten Gesellschaft privilegiert und unterstützt. Die Philosophin und Gendertheoretikerin Judith Butler [3] hat dieses System so treffend als „heterosexuelle Matrix“ bezeichnet. Die heterosexuelle und ciszentristische Matrix nimmt spezifisch in eine bestimmte Richtung ziehend Einfluss auf jede Interaktion. Wegen ihrer Omnipräsenz wird sie von den meisten Menschen nicht hinterfragt, jedoch von allen gespürt, mit dem Effekt, dass sie sich daran auszurichten versuchen. Wie alle permanent erlebten und beanspruchten Vorteile wird eine Privilegierung aufgrund der eigenen Heterosexualität oder der eigenen Cisidentität erst introspektionsfähig durch die empathische Erweiterung des Blickes auf die entsprechend deprivilegierten Personen und die sorgfältige Reflexion der eigenen gesellschaftlichen Position.

\section{„Wer etwas über Trans* wissen will, sollte Trans* fragen“[2].}

Trans* Personen empfinden, entscheiden und leben entgegen hetero- und ciszentristischen Vorannahmen [1]. Ihre geschlechtliche Selbstdefinition entspricht nicht den Fremdzuweisungen. Um von anderen in ihrem Identitätsgeschlecht angesprochen werden zu können, entscheiden sich manche trans* Personen für eine genderneutrale oder eine geschlechterkonnotierte Anrede, die sich nicht mit ihrem Zuweisungsgeschlecht deckt. Manche trans* Personen gestalten ihren Körper durch Binder oder Packer, durch lange Haare und/oder sorgfältige Rasur so, dass der Körper nicht ihr Zuweisungsgeschlecht verrät. Manche trans* Personen entscheiden sich für intensivere geschlechtsangleichende Maßnahmen, wie z. B. geschlechtsangleichende Operationen oder Hormoneinnahmen. Manche ändern ihren Vornamen und manche auch ihren amtlichen Geschlechtseintrag mittels aufwändiger juristischer Verfahren nach dem Namensänderungsgesetz oder dem „Transsexuellengesetz“ (TSG). Im Rahmen dieser Verfahren und der Beantragung von geschlechtsangleichenden medizinischen Behandlungen müssen sie sich ihre empfundene Geschlechtlichkeit von Gesundheitsprofessionellen bestätigen lassen, obwohl die Geschlechtsidenti- tät einer Fremddiagnostik gar nicht zugänglich ist [4]. Die konsultierten Gesundheitsprofessionellen begeben sich in diesem Prozess vielfach in die ethisch höchst problematische Position eines gatekeepings [2,5]. Das heißt von ihrer Entscheidung - und nicht der Selbstbestimmung der trans* Person - hängt ab, ob die Person die für ihre Transition ${ }^{2}$ dringlich benötigten medizinischen und rechtlichen Hilfen erlangt. Insbesondere trans* Personen, die als geschlechtlich uneindeutig gelesen werden, werden vielfach und biografisch durchgängig diskriminiert und angegriffen mit dem Ziel, sie zu einer geschlechtlich eindeutigeren Selbstpräsentation zu zwingen. Durch die Angriffe wird das Leid erzeugt, für welches dann wiederum das Gesundheitssystem zur Heilung angerufen wird.

Trans* Personen benötigen an manchen Stellen die Unterstützung des Gesundheitssystems, um ihren Körper ihrer tief empfundenen Geschlechtsidentität angleichen zu können, und/oder die gesundheitlichen Folgen von Diskriminierungen und Gewalt überwinden zu können. Auch benötigen sie das Gesundheitssystem in allen Belangen, in denen auch cisidente Menschen dieses in Anspruch nehmen: zur Behandlung von Erkrankungen, zur Unterstützung in der Schwangerschaft, zur Prävention uvm. Der Weltärztebund [7] hat sich unterdessen der Selbstwahrnehmung transgeschlechtlicher Menschen angeschlossen und beschreibt Transgeschlechtlichkeit seit 2015 als nicht-pathologische Variante der Geschlechtsidentität. Diese durch Aktivist*innen im Gesundheitssystem mühsam erkämpften Position ist auch der Ausgangspunkt des vorliegenden Artikels.

\section{Positionierung}

In den Gesundheitswissenschaften gibt es keinen „neutralen“ Standpunkt zum Thema „Trans* “ [8]. Jede*r begegnet dem Thema mit einem durch gesellschaftliche Konstruktionen durchzogenen spezifischen Erfahrungshintergrund, der auch wiederum prägt, was wir sehen, was wir für „Fachwissen“ halten, und wie wir einander in den Rollen als „Professionelle“, „Klient*in“ und „Forscher*in“ begegnen $[9,10]$. Jedoch eröffnet auch eine sorgfältige Reflexion der eigenen Erfahrungen nur einen begrenzten Horizont. Wie in anderen Bereichen besteht auch hier die Gefahr, sich zu schnell von einer zuhörenden Haltung wegzuwenden, aus dem Gedanken heraus, schon genug zu wissen.

\section{Diskriminierung und Gewalt}

Trans* Personen bilden keine einheitliche Gruppe. Unter ihnen befinden sich relativ privilegierte und ressourcenreiche Personen mit

2 Mit „Transition“ wird der Weg bezeichnet, den eine trans* Person geht, um ihre Geschlechtlichkeit ausdrücken und leben zu können. Im Rahmen der Transition kann sich die Person für verschiedene soziale (z. B. Comingout/ Selbstöffnung), rechtliche (z. B. Namensänderung) und medizinische (z.B. Hormontherapie, Epilation, geschlechtsangleichende Operationen) entscheiden. Trans* Personen, die sich dabei entscheiden, ihre Transgeschlechtlichkeit nicht offen leben zu wollen, leben „stealth“. Mit passing werden die Möglichkeiten einer trans* Person bezeichnet, im Identitätsgeschlecht eindeutig gelesen zu werden. Bezeichnungen für Schritte in der Transition, die suggerieren, die Person würde von einem Zustand in einen anderen wechseln und damit die Kontinuität des Entwicklungsprozesses verschleiern, wie z.B. das Wort „Geschlechtsumwandlung“ werden dem Selbstempfinden vieler Personen in der Transition nicht gerecht und deshalb zurückgewiesen. Für weitere im Themenfeld wichtige Bezeichnungen wird auf das Glossar des VLSP* e.V. verwiesen [6] 
vielen Möglichkeiten, sich in diskriminierenden Situationen sichtbar zu machen und zur Wehr zu setzen, jedoch auch hoch vulnerable Personen [11]. Diskriminierungen und Gewalt gegen trans* Personen stellen eine Form der gruppenbezogenen Menschenfeindlichkeit [12] dar. Sie äußern sich in negativen Einstellungen gegenüber trans* Personen, in Verachtung, in der Verweigerung von Unterstützung, im Vorenthalten von respektvollen und gerechtem Umgang, durch zudringliche Fragen, Zwangsouting, Beleidigungen und Beschimpfungen und in körperlichen Angriffen. Ziel der Angriffe ist die Errichtung einer Hierarchie, in der trans* Personen gewaltsam eine untergeordnete Position zugewiesen wird. Diese zugewiesene Position von trans* Personen legitimiert wiederum aus der Perspektive der Angreifer*innen weitere Diskriminierungen und Gewalt. Die Gewaltfolgen werden der betroffenen trans* Person zugeschrieben. Die Gesellschaft wiederum beschneidet u. a. durch Ermöglichung diskriminierenden Verhaltens in Polizei und Behörden die Möglichkeit der angegriffenen Menschen, sich zu wehren $[13,14]$.

Als Bereiche mit den höchsten Diskriminierungsraten zeigen sich in deutschen und europäischen Untersuchungen die Herkunftsfamilien und Schulen, der öffentliche Raum und Ämter, Behörden und das Gesundheitswesen. Dementsprechend sind viele trans* Personen in diesen Bereichen nicht out (das heißt, sie öffnen sich nicht in Bezug auf ihre Transgeschlechtlichkeit). Besonders stark betroffen von trans* feindlicher Diskriminierung und Gewalt sind transweibliche Personen und trans* Personen, die zusätzlich z. B. von Rassismus betroffen sind, oder in Armut aufwachsen und leben, oder obdachlos sind [5]. Die Folgen von Diskriminierungen und Gewalt werden von der Schwere und Häufigkeit der Erfahrungen geprägt, von der Vulnerabilität der Person, den Reaktionen des Umfelds, sowie von den Möglichkeiten, sich zur Wehr zu setzen und Unterstützung zu erhalten.

Die europaweit durchgeführte Untersuchung der Europäischen Grundrechteagentur [15] mit 93079 lesbischen, schwulen, bisexuellen und trans* Teilnehmenden zeigte, dass $47 \%$ aller befragten LSBT*-Personen innerhalb des letzten Jahres Diskriminierung oder Belästigung aufgrund ihrer sexuellen Ausrichtung und oder Genderidentität erfahren haben. $26 \%$ waren in den letzten fünf Jahren von Angriffen oder Gewaltandrohungen betroffen. $80 \%$ erinnern sich an negative Bemerkungen oder Mobbing gegenüber jugendlichen Lesben, Schwulen, Bisexuellen oder trans* Personen in der Schule. Durchgängig berichteten in dieser Untersuchung trans* Personen von einem besonders hohen Ausmaß an Diskriminierungs- und Gewalterfahrungen. Die Diskriminierungen und Gewalt in Herkunftsfamilien und Schule können neben den unmittelbaren gesundheitlichen Gewaltfolgen zu Brüchen in der Bildungsbiografie führen. Viele trans* Jugendliche versuchen sich vor Gewalt in der Schule zu schützen, indem sie die Schule nicht mehr besuchen und Kontakt zu anderen marginalisierten Jugendlichen aufnehmen $[13,16]$.

Auf dem Arbeitsmarkt erzielen trans* Personen ein relativ geringes Einkommen, auch wenn sie über eine hohe Qualifikation verfügen, weil sie vermehrt unterhalb ihres Qualifikationsniveaus beschäftigt werden. Sie werden bei der Arbeitsvermittlung benachteiligt und sind vermehrt von Arbeitslosigkeit, Kündigung und Armut betroffen [16, 17].

Aus dem Gesundheitssystem berichtet in der Untersuchung der Europäischen Grundrechteagentur [15] ungefähr ein Fünftel der befragten trans* Personen von unangemessenem Verhalten von Gesundheitsversorger*innen, z. B. in Form von ungerechtem Verhalten, belästigender Neugier, Ignoranz gegenüber ihren Gesundheitsbedarfen. Viele trans* Personen vermeiden aus Angst notwendige Behandlungen oder müssen nach Diskriminierungen ihre Gesundheitsversorger*innen wechseln. Derzeitig affirmiert die Aufführung von „Transsexualismus“ als Diagnose F64.0 in dem Kapitel „Persönlichkeits- und Verhaltensstörungen“ im ICD-10 [18] noch die diskriminierende gesellschaftliche Perspektive, Transgeschlechtlichkeit sei etwas, was sich nicht mit psychischer Gesundheit vereinbaren ließe und auf dessen Prävention und Beseitigung sich die Bemühungen des Gesundheitssystems zu richten hätten [19]. Die Verfahren zur Kostenübernahme für die von vielen trans* Personen benötigten Transitionsbehandlungen greifen tief in das Selbstbestimmungsrecht der betreffenden Personen ein, kosten viel Mühe und Lebenszeit. Die als Bedingung für die Kostenübernahme für die Hormonbehandlung, die Epilation und die geschlechtsangleichenden Operationen gesetzte Psychotherapie stellt strukturell eine Zwangsmaßnahme dar, die das Vertrauen und die Möglichkeiten zur Selbstöffnung in der verordneten Therapie nachhaltig beeinträchtigen. Ein Sechstel von 98 in Nordrhein-Westfalen befragten trans* Personen [20] war dementsprechend mit der Psychotherapie, dem Begutachtungsverfahren im Rahmen der Kostenübernahmeentscheidungen für die benötigten Transitionsoperationen unzufrieden. Benennt eine trans* Person in der Psychotherapie Substanzgebrauch, muss sie befürchten, dass sie nicht die Indikation für die von ihr benötigten Transitionsbehandlungen erhält [21].

Diskriminierungen und der Zugang zu Ressourcen überschneiden sich in einem intersektionalen Bedingungsgefüge [11, 22]. Arme und psychisch kranke trans* Personen haben gleichzeitig größere Schwierigkeiten, sich gegen Diskriminierungen im Gesundheitssystem zu wehren und finden seltener Versorger*innen, die bereit sind, sie mit Respekt zu behandeln.

Ein Beispiel für Diskriminierung im Gesundheitssystem:

Eine transfeminine Person entscheidet sich zur Behandlung in einer psychosomatischen Klinik. Sie wird auf Station aufgenommen. Als sie ihre Transgeschlechtlichkeit kommuniziert, entsteht beim Personal eine für die Patientin sichtbare Verwirrung. Obwohl die Patientin von Mitpatient*innen zuvor freundlich und respektvoll aufgenommen worden ist, teilt ihr die zugewiesene Therapeutin mit, ihr Genital stelle eine Gefahr für ihre Mitpatient*innen da. Vor der geschlechtsangleichenden Operation sei sie nur als eine „Art Frau“ anzusehen.

\section{Epidemiologie schädlichen Substanzgebrauchs bei trans* Personen in Deutschland}

Schädlicher und insbesondere süchtiger Gebrauch von psychoaktiven Substanzen zeigen sich in der epidemiologischen Forschung auch als Marker gesellschaftlicher Stigmatisierung, Ausgrenzung und Gewalt $[5,23,24]$. Dies gilt insbesondere für den Konsum illegalisierter psychoaktiver Substanzen. 
In Deutschland verfügen wir derzeitig nicht über hinreichend ökologisch valide Daten zum Substanzgebrauch von trans* Personen. Dies liegt zum einen daran, dass trans* Lebensweisen in bevölkerungsstatistischen Untersuchungen zur Gesundheit in Deutschland bislang nicht erfasst worden sind und auch an der noch unzureichenden Berücksichtigung intersektionaler Aspekte in der deutschsprachigen Forschung zur Trans*-Gesundheit. Aktuell verfügen wir in Deutschland fast ausschließlich über Gesundheitsdaten von trans* Personen, die die Angebote von medizinischen Transitionsbehandlungen in dafür spezialisierten Institutionen in Anspruch nehmen. Aus einer Stichprobe von insgesamt 305 Teilnehmer*innen, die in den Niederlanden, Belgien, Deutschland (Hamburg) und Norwegen geschlechtsangleichende medizinische Therapien in darauf spezialisierten Kliniken suchten, wiesen im Untersuchungszeitraum (2007-2010) von den 53 Teilnehmer*innen in Deutschland 8 \% psychische Störungen durch den Konsum psychotroper Substanzen auf. $11 \%$ berichteten von solchen Störungen im Verlauf ihres Lebens. Eine Essstörung im Untersuchungszeitraum gaben $2 \%$ an. Die Lebenszeitprävalenz einer Essstörung lag bei $4 \%$ [25]. Vergleichen wir diese Zahlen mit einer repräsentativen Bevölkerungsstichprobe aus der Upper Bavarian Study zusammengefasst in [26], in der u. a. der schädliche Gebrauch und die Abhängigkeit von Alkohol und Drogen untersucht wurden und dafür eine SechsMonats-Prävalenz von insgesamt 5,8\% konstatiert wurde, so zeigen sich die Prävalenzzahlen für schädlichen und abhängigen Substanzgebrauch bei den Behandlungssuchenden von geschlechtsangleichenden medizinischen Maßnahmen nur leicht erhöht. Zu Essstörungen lassen sich aufgrund der geringen Fallzahl keine vergleichenden Angaben machen.

Deutlich besser untersucht ist das Thema in den USA, sowohl, was die Anzahl der entsprechenden Arbeiten betrifft, als auch deren Qualität [vergl. Vogt in diesem Heft und 27]. Die US-amerikanischen Daten [z. B. [28]] sprechen für eine erhöhte Prävalenz für substanzassoziierte psychische Störungen bei trans* Personen im Vergleich zu einer allgemeinen Bevölkerungsstichprobe. Dabei sind trans* Personen, die zusätzlich aufgrund eines anderen Merkmals marginalisiert sind, z. B. weil sie obdachlos leben, arm oder von Rassismus betroffen sind, nochmal verstärkt von Substanzkonsumstörungen betroffen, ebenso Menschen, die mit ihnen zusammenleben oder ihnen nahestehen [vergl. 5, 29]. Die Daten aus den USA lassen sich wegen der unterschiedlichen Gestaltung des Gesundheitssystems und der größeren Sichtbarkeit von trans* Personen dort mit den entsprechenden soziokulturellen Konsequenzen nur eingeschränkt auf Deutschland übertragen.

\section{Dynamiken schädlichen Substanzgebrauchs bei marginalisierten Personen}

Stigmatisierungen, Diskriminierung und Gewalt beeinträchtigen die psychische und körperliche Gesundheit der betroffenen Menschen [25]. Finden Diskriminierungen im Gesundheitssystem statt, sind das Barrieren, die nur schwer zu überwinden sind. Da die Konsumstörungen aus diesen Gründen oft nicht frühzeitig behandelt werden, kann das dazu führen, dass die Erkrankungen chronifizieren [3032]. Diskriminierungen und Gewalt setzen die davon betroffenen
Menschen einer Vielzahl unangenehmer und zum Teil auch unerträglicher Empfindungen in Form von Ängsten, Wut, Hypervigilanz, Scham ... aus. Das aus der Gewalt resultierende seelische und körperliche Leiden, das Abdrängen in die soziale Isolation, das Misstrauen der Betroffenen wird vom diskriminierenden Umfeld wiederum den gewaltbetroffenen Menschen als Wesensmerkmal zugeschrieben, woraus dann eine Verschärfung der Stigmatisierung resultiert.

Erfahrungen mit Stigmatisierungen und Gewalt machen Substanzgebrauch als Bewältigungsstrategie verstehbar. Substanzgebrauch kann der Regulation unerträglicher Gefühle dienen, z. B. wenn wegen der Diskriminierungen die Zukunftschancen massiv begrenzt werden. Eine Essstörung kann durch aktive Einflussnahme auf die Körperform ein genderdysphorisches Erleben regulieren [33]. Der unmittelbar als erleichternd empfundene Effekt des Substanzgebrauchs und die Suchtdynamik erschweren den Ausstieg aus schädlichen Substanzgebrauchs- und Verhaltensmustern [21]. Substanzgebrauch dient auch der Regulierung von Nähe und unterbindet potenziell schädigenden Kontakt [21]. Diese Schutzstrategien schwächen jedoch auch das Behandlungsbündnis, wenn eine suchtbetroffene Person Therapie aufsucht.

Verschiedene Modelle versuchen, den Zusammenhang zwischen gesellschaftlicher Ausgrenzung und Beeinträchtigungen des gesundheitsbezogenen Verhaltens konzeptuell und nicht-diskriminierend zu fassen, z. B. das Minority-Stress-Modell von Ilan Meyer [34] und die Objectification-Theory [35] ${ }^{3}$.

2013 publizierte Sevelius eine trans* spezifische Ausarbeitung von marginalisierungsbedingten Gesundheitsfolgen und legte im Modell gleichzeitig die Möglichkeiten und Chancen einer transspezifischen Gesundheitsversorgung dar. Das von Sevelius aufgrund von Interviews mit transweiblichen People of Color entwickelte Genderaffirmation Framework beschreibt, wie die gesellschaftliche Unterdrückung transgeschlechtlicher Lebensweisen sich in einer Internalisierung des Stigmas in Form von Selbstentwertungsprozessen und Ängsten niederschlägt. Die daraus resultierenden Belastungen verstärken einerseits die innere Notwendigkeit in der Person, sich um soziale Anerkennung durch Maßnahmen für ein verbessertes passing im Identitätsgeschlecht zu bemühen, um die Ausgrenzung zu mildern, gleichzeitig blockieren die Diskriminierungen den Zugang zu geschlechtsangleichenden Maßnahmen durch die Errichtung von Versorgungsbarrieren. Die gesellschaftlichen Ausgrenzungen bringen die betroffene trans* Person damit in ein schier unlösbares Dilemma, wenn sie sich bei der Suche nach den dringlich benötigten Transitionsmaßnahmen im durch Diskriminierungen vulnerabilisierten Zustand einer Gesundheitsversorgung anvertrauen muss, in der ihr wiederum erneut respektlos begegnet wird. Um diesem Dilemma auszuweichen, bieten sich gesundheitsschädigende Handlungen erst mal an, um die Belastung zu reduzieren, z. B. Abdämpfen der Wahrnehmung für Verletzungen durch Substanzgebrauch, Besorgen von Hormonpräparaten auf dem freien Markt unter Umgehung einer endokrinologischen

3 Das Minority-Stress-Modell wurde ursprünglich zur Konzeptualisierung gesundheitsbezogenem Verhaltens von Lesben und Schwulen entwickelt. Eine Adaptation auf die Situation von trans* Personen wurde u.a. von Güldenring [36] vorgelegt. Sowohl das Minority-Stress-Modell als auch die Objectification-Theory eignen sich hervorragend für die Therapieplanung und die Psychoedukation in der Therapie und sind ausführlich beschrieben in Wolf [21]. 
Behandlung und ohne Blutbildkontrollen und selbstverletzendes Verhalten als Ausdruck der empfundenen Perspektivlosigkeit. Die qualitativen Daten von Sevelius [37] legen nahe, dass der Zugang zu Transitionsmaßnahmen Depressivität und damit auch den Drang Alkohol zu konsumieren verringert.

\section{Suchtbehandlung von trans* Personen}

Trans* Personen, die in einem selbst- und/oder fremdschädigenden Ausmaß psychotrope Substanzen konsumieren oder unter einer Essstörung leiden, benötigen ein therapeutisches Angebot, welches ihre spezifischen Lebenserfahrungen mitbedenkt und es ihnen so ermöglicht, eine suchttherapeutische Behandlung als hilfreich und sinnvoll zu begreifen.

Hierfür ist wesentlich, dass die Behandlung eine Struktur zur Verfügung stellt, die tragend und sicher genug ist, um sich den Erfahrungen von Entzug und Entwöhnung stellen zu können. In allen Institutionen der Suchttherapie sollte das Wissen verankert werden, dass es trans* Personen gibt. Sobald eine Institution mehr als 200 Klient*innen behandelt, ist es statistisch hochwahrscheinlich, dass auch trans* Personen dabei sind, auch wenn diese evtl. Gründe sehen, nicht sichtbar zu werden $[38,39]$. So sollte sich jedes Team in der Suchthilfe fragen, wenn bisher vermeintlich keine trans* Personen die Institution genutzt haben, woran das liegt und wie hier Möglichkeiten geschaffen werden können, dass trans* Personen die Institution für sich als Ort identifizieren können, an dem sie offen auftreten können. In diesem Zusammenhang empfiehlt es sich im Sinne einer Fehlerkultur [40] nach stattgefundenen Diskriminierungssituationen, die trans* Klient*innen evtl. in der Institution erlitten haben, zu fragen, und die Gründe für Therapieabbrüche auch dahingehend zu untersuchen, ob Diskriminierungen dahintergestanden haben könnten. Diskriminierungen durch Kolleg*innen sollten transparent und konsequent begegnet werden. Nur wenn die Bedingungen für eine Sichtbarkeit von trans* Personen strukturell diskriminierungssicher gestaltet werden, können trans* Personen für sich die Behandlungseinrichtung nutzen, ohne sich verstecken zu müssen.

Eine durchgängige Ansprache und Behandlung von trans* Personen im jeweiligen Identitätsgeschlecht sollte selbstverständlich sein. Dies gilt unabhängig davon, welche Schritte eine trans* Person in der sozialen und medizinischen Transition hat gehen wollen oder können [1, 29]. Hierzu gehört auch, mit der Person zu klären, in welchem Kontext sie wie angesprochen werden möchte. In allen verwendeten Fragebögen, die zur Klärung der psychischen Belastungssymptomatik eingesetzt werden, sollte eine Anrede entsprechend des Identitätsgeschlechts möglich gemacht werden. Auch in Anträgen, Stellungnahmen und Berichten empfiehlt sich die Verwendung der Anrede, die die Person wünscht.

Eine Suchtbehandlung braucht grundlegend eine stabile, transparente und respektvolle Gestaltung der therapeutischen Beziehung [41]. Personen mit einer transrespektvollen Haltung zu begegnen bedeutet, den Wert, die Würde und den gesellschaftlichen Beitrag von Personen, deren Gender-Identität oder Gender-Ausdruck den ihnen permanent entgegengebrachten Erwartungen nicht entspricht, anzuerkennen [14,42]. Diese Anerkennung findet Ausdruck in der konkreten Gestaltung der therapeutischen Kommunikation. Diese geht auf der Basis einer transrespektvollen Haltung auch komplementär auf die Funktionalität von Substanzgebrauch bei marginalisierten Menschen ein. Wenn jemand konsumiert, um sich aus unsicheren, weil diskriminierenden und gewaltvollen, Kontakten zurückziehen zu können, muss in der Therapie der Kontakt sicher und diskriminierungssensibel gestaltet werden. Rückfallauslösend sind bei trans* Personen oft Ängste, Diskriminierungen schutzlos gegenüber zu stehen, Einsamkeit, eine Überwältigung durch belastende Gefühle in einem gewaltvollen Klima oder durch Flashbacks nach Traumatisierungen, sowie durch Diskriminierungen erzeugte Perspektivlosigkeit. Die Scham durch die Erfahrungen des Kontrollverlusts in der Suchterkrankung verstärkt die Scham durch die Zuweisung einer gesellschaftlich inferioren Position und errichtet Kommunikationsbarrieren, die validierend angesprochen werden müssen, um sie abbauen zu können.

Angesichts der hohen Diskriminierungs-, Marginalisierungs- und Gewaltbetroffenheit von trans* Personen sollten Suchtbehandlungen für trans* Personen die Möglichkeiten eines traumaspezifischen therapeutischen Angebots beinhalten [27, 43].

\section{FAZIT FÜR DIE PRAXIS}

Als Gesundheitsversorger*innen sind wir dazu aufgerufen, uns aktiv dafür einzusetzen, Gesundheitsangebote so zu gestalten, dass sie zu sicheren und diskriminierungssensiblen Orten für trans*Personen werden [5, 7, 44]. Es liegt ausdrücklich nicht in der Verantwortung der trans* Personen, die uns in der Klient*innenrolle gegenübertreten, dafür zu sorgen, dass sie sich nicht durch Diskriminierungen berühren lassen. Auch sollten wir als Professionelle trans* Klient*innen nicht in die Position bringen, erst Aufklärungsarbeit leisten zu müssen, bevor sie ein Gesundheitsangebot nutzten. Die Chancen, dass ein Gesundheitsangebot von trans*Personen und auch anderen marginalisierten Personen als hilfreich wahrgenommen werden kann, hängen ab von einer konsequenten und strukturell verankerten antidiskriminatorischen Arbeit in einer Praxis oder Klinik und im Kompetenzaufbau bezogen auf spezifische Versorgungsbedarfe dieser Personen. Wir wissen leider noch zu wenig über das Outcome von Suchtbehandlungen von trans* Personen. So braucht es, um im Suchthilfebereich mit trans* Klient*innen auch evidenzbasiert arbeiten zu können, mehr partizipative Forschung, die trans* Personen bei Forschungskonzeptionen Entscheidungskompetenzen zuweist [vergl. 45]. Für wichtig halte ich in diesem Zusammenhang zudem Forschung, die untersucht, inwieweit sich medizinische Maßnahmen zur Unterstützung in der Transition auch symptomlindernd auf eine Suchtdynamik auswirken können. Aus der Praxis sind Verläufe bekannt, in denen die Perspektiverweiterung durch die Ermöglichung einer Selbstöffnung als trans*und einer medizinischen Transitionsbehandlung mit einer Remission einer Suchterkrankung einhergegangen sind. Wir brauchen in der Suchttherapie mehr Wissen, um trans* Personen gut behandeln zu können. Aber: „What we do know ... is that the majority of people who desire to transition, will improve after they transition“ [5, o.S.]. 


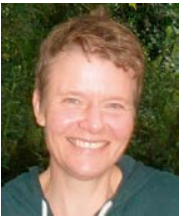

\section{Dr. Gisela Wolf}

arbeitet in Berlin als psychologische* Psychotherapeut*in

überwiegend mit Personen aus Isbtq-Communities.

\section{Interessenkonflikt}

Der die Autor* gibt an, dass kein Interessenkonflikt besteht.

Literatur

[1] Wolf G, Meyer E. Sexuelle Orientierung und Geschlechtsidentität - [ $k$ ] ein Thema in der Psychotherapie? Psychotherapeutenjournal 2017; 2: 130-139Im Internet https://www.psychotherapeutenjournal.de/ptk/ web.nsf/gfx/38CB16EDB11EE29BC125813F0024E282/\$file/ ptj_2017-2.pdf; Stand: 15.02.2018

[2] Hamm J, Sauer A.Selbstbestimmung und neue Sichtweisen auf Trans* - wer hat Angst vorm Perspektivwechsel [2012]. Im Internet: www. forum. sexualaufklaerung.de/index.php?docid $=1679$ Stand: 05.02.2018

[3] Butler J. Das Unbehagen der Geschlechter. Frankfurt/M.: Suhrkamp; 1991

[4] Güldenring A. Zur „Psychodiagnostik von Geschlechtsidentität“ im Rahmen des Transsexuellengesetzes. Zeitschrift für Sexualforschung 2013; 26: 160-174

[5] Graham J.Guidelines for Psychosocial Assessments for Sexual Reassignment Surgery or Gender Affirmation Surgery [2013]. Im Internet: www.sfdph.org/dph/files/THS/TransgenderHealthServices_ MH\%20Guidelines.pdf; Stand 25.01.2018

[6] Wolf G, Fünfgeld M, Oehler R et al.Empfehlungen zur Psychotherapie und Beratung mit lesbischen, schwulen und bisexuellen Klient_innen: Glossar [2015]. Im Internet: www.vlsp.de/files/pdf/zu_vpp1-15_s2_ empfehlungen_glossar.pdf; Stand: 16.11.2016

[7] World Medical Association (WMA). WMA Statement on Transgender People. Adopted by the 66th General Assembly, Moscow, Russia, Octobre 2015. Im Internet: https://www.wma.net/policies-post/ wma-statement-on-transgender-people/ Stand: 25.02. 2016

[8] Haraway D. Situated Knowledges: The Science Question in Feminism and the Privilege of Partial Perspective. Feminist Studies 1988; 14: 575-599

[9] Wolf G.Gendernonkonform. Transidente Kinder besser vor Schikanen schützen [2016]. Im Internet: http://www.tagesspiegel.de/berlin/ queerspiegel/gendernonkonform-transidente-kinder-besser-vor-schikanen-schuetzen/19167870.html Stand: 15.02.2018

[10] LaSala MC. When Interviewing "Family". Maximizing the Insider Advantage in the Qualitative Study of Lesbians and Gay Men. In: Meezan W, Martin JI, (Hrsg.). Handbook of Research with Lesbian, Gay, Bisexual, and Transgender Populations. New York, London: Routledge; 2009: 208-222

[11] Das Nair R, Butler C. Intersectionality, Sexuality and Psychological Therapies. UK, USA: British Psychological Society and John Wiley \& Sons/Blackwell; 2012

[12] Zick A, Hövermann A, Krause D. Die Abwertung von Ungleichwertigen. Erklärung und Prüfung eines erweiterten Syndroms der Gruppenbezogenen Menschenfeindlichkeit. In: Heitmeyer W, (Hrsg.). Deutsche Zustände. Folge 10. Berlin: Suhrkamp; 2012: 64-86
[13] LesMigraS [Antigewalt- und Antidiskriminierungs-Bereich der Lesbenberatung Berlin e.V. “...Nicht so greifbar und doch real”. Eine quantitative und qualitative Studie zu Gewalt- und [Mehrfach] Diskriminierungserfahrungen von lesbischen, bisexuellen Frauen und Trans* in Deutschland [2012]. Im Internet: http://lesmigras.de/tl_files/ lesmigras/kampagne/Dokumentation \%20Studie \%20web.pdf; Stand: 07.02.2018

[14] Transgender Europe [TCEU]. Balzer C.Transrespect versus transphobia worldwide. Terminology [2018]. Im Internet: http://transrespect.org/ en/working-definitions/ Stand: 07.02.2018

[15] European Union Agency For Fundamental Rights (FRA). Being Trans in the European Union. Comparative analysis of EU LGBT survey data. Luxembourg: Publications Office of the European Union; 2014

[16] Franzen J, Sauer A. Benachteiligung von Trans* Personen, insbesondere im Arbeitsleben. Expertise für die Antidiskriminierungsstelle des Bundes. Berlin: Antidiskriminierungsstelle des Bundes; 2010

[17] Whittle S, Turner L, Combs R et al.Transgender EuroStudy. Legal Survey and Focus on the Transgender Experience of Health Care. Brüssel: ILGA-Europe [2008]. Im Internet: www.bizeps.or.at/news. php?nr $=8811$ Stand: 15.04 .2011

[18] Dilling H, Mombour W, Schmidt MH. Internationale Klassifikation psychischer Störungen ICD-10 Kapitel V [F], Klinisch-diagnostische Leitlinien. 6. AuflBern: Verlag Hans Huber; 2008

[19] Wolf G.Psychopathologisierungsprozesse von LSBT * I-Lebensweisen in DSM-5 und ICD 10, Kapitel F. [2013]. Im Internet: http://hirschfeldkongress.de/images/download/publikationen/Psychopathologisierungsprozesse \%20von \%20LSBT-I-Lebensweisen $\% 20$ in \%20DSM-5 \%20 und $\% 20$ ICD \%2010, \%20Kapitel \%20F \%20-\%20Gisela \%20Wolf.pdf Stand: 15.02 .2018

[20] Fuchs W, Ghattas DC, Reinert D et al.Studie zur Lebenssituation von Transsexuellen in Nordrhein-Westfalen. Ministerium für Gesundheit, Emanzipation, Pflege und Alter des Landes Nordrhein-Westfalen/ LSVD e.V [2012]. Im Internet: https://www.Isvd.de/fileadmin/pics/ Dokumente/TSG/Studie_NRW.pdf Stand: 15.02.2018

[21] Wolf G. Substanzgebrauch bei Queers. Dauerthema und Tabu. Göttingen: Wallstein-Verlag; 2017

[22] Crenshaw KM. Mapping the margins: intersectionality, identity politics, and violence against women of color. Stanford Law Review 1991; 43: 1241-1299

[23] Gunderson CL. Sucht - eine Fahrt im Definitionskarussell. In: Gahleitner SB, Gunderson CL, (Hrsg.). Frauen Trauma Sucht. Neue Forschungsergebnisse und Praxiserfahrungen. Kröning: Asanger Verlag; 2008: 101-111

[24] Russel M, Testa M, Wilsnack S. Alcohol use and Abuse. In: Goldman MB, Hatch MC, (Hrsg.). Women \& Health. San Diego, San Francisco, New York, Boston, London, Sydney, Tokyo: Academic Press; 2000: 589-598

[25] Heylens G, Elaut E, Kreukels BPC et al. Psychiatric characteristics in transsexual individuals: multicentre study in four European countries. BJPsych 2014; 204: 151-156. doi:10.1192/bjp.bp.112.121954

[26] Soyka M. Störungen durch Alkohol. In: Möller HJ, Laux G, Kapfhammer HP, (Hrsg.). Psychiatrie und Psychotherapie. Band 2. Berlin: Springer; 2008: 143-186

[27] Shelton M. Fundamentals of LSBT substance use disorders. Multiple identities, multiple challenges. New York: Harrington Park Press; 2017

[28] Institute of Medicine. The Health of Lesbian, Gay, Bisexual, Transgender People: Building a Foundation for Better Understanding. Washington DC: The National Academies Press; 2011

[29] Wolf ECM, Dew B. Understanding Risk Factors Contributing to Substance Use Among MTF Transgender Persons. Journal of LGBT Issues in Counseling 2012; 6: 237-256 
[30] Shelgrove JW, Jasudavisius AM, Rowe BW et al. "Completely out-atsea" with "two-gender medicine". A qualitative analysis of physicianside barriers to providing healthcare for transgender patients. BMC Health Service Research 2012, [12] doi:10.1186/1472-6963-12-110

[31] Wolf G, Schleider K, Pfarrherr K. Die Qualität der Versorgung von Menschen mit psychischen Beeinträchtigungen in Netzwerken professioneller Gesundheitsdienste. Zeitschrift für Qualitative Forschung [ZQF] 2010; 11: 91-110

[32] Wolf G. Gesundheitsversorgung: Barrieren für homosexuelle Patienten. Dtsch. Arztebl. 2010; 107:[44]: A-2166/B-1876/C-1851. Im Internet: www.aerzteblatt.de/archiv/79047/GesundheitsversorgungBarrieren-fuer-homosexuelle-Patienten Stand: 15.02.2018

[33] Hepp U, Milos G. Gender identity disorder and eating disorder. Int J Eat Disord 2002; 32: 473-478

[34] Meyer I. Minority stress and mental health in gay men. Journal of Health Sciences and Social Behavior 1995; 36: 38-56

[35] Fredrickson BL, Roberts TA. Objectification Theory. Toward Understanding Women's Lived Experiences and Mental Health Risks. Psychol Women Q 1997; 21: 173-206

[36] Güldenring A. Phasenspezifische Konfliktthemen eines transsexuellen Entwicklungsweges. PID 2009; 1: 25-31

[37] Sevelius JM. Gender Affirmation: A Framework for Conceptualizing Risk Behavior among Transgender Women of Color. Sex Roles.2013: 1: 68 675-689 doi:10.1007/s11199-012-0216-5.eutsch M Guidelines for the Primary and Gender-Affirming Care of Transgender and Gender Nonbinary People. San Francisco: University of California; 2016.
[38] Güldenring A, Sauer A. Trans * ...inklusiv? Geschlechtsidentitäten in Medizin, Recht und Gesellschaft. In: Diehl E, (Hrsg.). Teilhabe für alle?! Lebensrealitäten zwischen Diskriminierung und Partizipation. Bundeszentrale für politische Bildung. Schriftenreihe Band 10155. Bonn: 2017: 231-257

[39] Paula H. Patientensicherheit und Risikomanagement in der Pflege. Für Stationsleitungen und PDL. 2., überarb. AuflHeidelberg: Springer; 2017

[40] Norcross JC. Empirically Supported Therapy Relationships. In: Norcross JC, (Hrsg.). Psychotherapy Relationships That Work. Therapist Contributions and Responsiveness to Patients. New York: Oxford University Press; 2002: 3-16

[41] Günther M.Psychotherapeutische und beratende Arbeit mit Trans* Erfahrung, Haltung, Hoffnung; 2015. Im Internet: www.vlsp.de/files/ pdf/vpp1-15_s7_trans.pdf Stand: 15.02.2018

[42] Gahleitner SB, Gunderson C. Frauen Trauma Sucht. Neue Forschungsergebnisse und Praxiserfahrungen. Kröning: Asanger Verlag; 2008

[43] American Psychological Association (APA). Guidelines for Psychological Practice With Transgender and Gender Nonconforming People. American Psychologist, 2015; Dec. Im Internet: www.apa.org/practice/ guidelines/transgender.pdf Stand: 29.03. 2016

[44] Wright M, Block M, Unger H. Stufen der Partizipation in der Gesundheitsförderung. Dokumentation 13. Bundesweiter Kongress Armut und Gesundheit - 30. November/ 1. Dezember [2007]. Im Internet: www.armut-und-gesundheit.de/uploads/tx_gbbkongressarchiv/ Wright__M..pdf Stand: 07.02.2018 